\title{
Prevalence of Tuberculosis by GeneXpert Method in Karnali Academy of Health Sciences Teaching Hospital, Jumla, Nepal
}

\author{
Bibek Pun Magar ${ }^{1, \text { * }, \text { Niresh Thapa }}{ }^{2}$, Elisha Rana ${ }^{3}$, Keshav Raj Joshi ${ }^{4}$, Narayani Maharjan ${ }^{5}$, \\ Binita Bista ${ }^{6}$ \\ ${ }^{1}$ Department of Clinical Biochemistry, Karnali Academy of Health Sciences, Jumla, Nepal \\ ${ }^{2}$ Department of Emergency, Karnali Academy of Health Sciences, Jumla, Nepal \\ ${ }^{3}$ Karnali Academy of Health Sciences, Jumla, Nepal \\ ${ }^{4}$ Krown Laboratory, Kathmandu, Nepal \\ ${ }^{5}$ Program \& Department of Clinical Laboratory Medicine, Center for Gene Diagnosis, Zhongnan Hospital of Wuhan University, Wuhan, China \\ ${ }^{6}$ Department of Nursing, District Health Office, West Rukum, Nepal
}

\author{
Email address: \\ bibekpunmagar9@gmail.com (B. P. Magar) \\ ${ }^{*}$ Corresponding author
}

\section{To cite this article:}

Bibek Pun Magar, Niresh Thapa, Elisha Rana, Keshav Raj Joshi, Narayani Maharjan, Binita Bista. Prevalence of Tuberculosis by GeneXpert Method in Karnali Academy of Health Sciences Teaching Hospital, Jumla, Nepal. Biomedical Sciences. Vol. 6, No. 3, 2020 , pp. 56-60. doi: $10.11648 /$ j.bs.20200603.13

Received: July 27, 2020; Accepted: August 8, 2020; Published: August 20, 2020

\begin{abstract}
Tuberculosis (TB) is a communicable disease that is a major public health problem and one of the top 10 causes of death worldwide. Globally, an estimated 10 million people fell ill with TB in 2018 . The population of Nepal accounts for $0.45 \%$ of the global TB cases. GeneXpert is a molecular test method for TB which detects the presence of Mycobacterium tuberculosis, as well as tests for resistance to drug rifampicin. The study aimed to find the prevalence of TB in the rural areas of Karnali province, Jumla. This retrospective cross-sectional study was done in Karnali Academy of Health Sciences Teaching Hospital (KAHS-TH), Jumla. Required data was retrieved from the Laboratory record and medical record section. The data was collected and analyzed by SPSS version 16. Altogether 805 individuals were screened from December 2017 to May 2020. Among them, $96(11.9 \%)$ were infected with TB in which $4(4.16 \%)$ were rifampicin-resistant. Out of the total, 457 males screened for TB where $67(14.66 \%)$ were positive for TB in which $2(2.98 \%)$ were rifampicin-resistant and among 348 females screened 29 $(8.33 \%)$ were positive for TB in which $2(6.89 \%)$ were rifampicin-resistant. The highest prevalence of $20.63 \%$ was observed in the age group of 21-30 years. The study suggests TB infection is lower in Jumla as compared to the overall prevalence of Nepal and helps to identify the high-risk group of TB infection. It also implies increasing the GeneXpert test for a large population, which may help in future TB control programs in Jumla and similar geographical area.
\end{abstract}

Keywords: GeneXpert, Prevalence, Rifampicin-Resistant, Tuberculosis

\section{Introduction}

Tuberculosis (TB) is one of the deadliest common infectious diseases in developing countries. It is usually caused by Mycobacterium tuberculosis $(M . t b)$, a bacterium which generally affects the lungs, but also affects other body parts. The diagnosis of active TB is based on chest X-rays, along with microscopic examination, molecular tests, and culture of body fluids. [1]

According to the World Health Organization (WHO), a total of 1.5 million died from TB in 2018 (including 251000 people with HIV). [2] Global tuberculosis report 2017 (WHO) report $4.1 \%$ and $19 \%$ of newly diagnosed and previously treated TB patients, respectively, were multi-drug resistant tuberculosis (MDR-TB).[3] In 2019, data were reported by 202 countries and territories that account for more than $99 \%$ of the world's population and the estimated number of TB cases. More than 
$95 \%$ of deaths occurred in developing countries. [4]

In Nepal, an estimated 45,000 people fell ill with TB in 2018, only 32,474 cases were reported. Additionally, drug-resistant TB continues to pose a dangerous health threat to our populations. [5]

The GeneXpert MTB/RIF is a new cartridge-based nucleic acid amplification test (NAAT) for the rapid diagnosis of TB and drug resistance. [6] The test simultaneously detects Mycobacterium tuberculosis (MTB) complex DNA and resistance to rifampicin (RIF) in less than 2 hours. [6] This test method is more sensitive than sputum microscopy in detecting TB, and it has similar accuracy as culture. [7]

In Nepal, GeneXpert test was introduced in December 2011, while KAHS-TH, Jumla started GeneXpert test from December 2017 which is helping in prompt diagnosis as well as testing resistance to rifampicin in TB infected individuals. Although Karnali province has the lowest prevalence in Nepal, though with the introduction of GeneXpert test more and more cases are emerging which may have been missed by routine conventional methods helping us to reach the true estimate of TB in the area and as well as planning for future in controlling and treatment of TB. Hence, the study aimed to access the prevalence of TB by GeneXpert method in this rural area of Nepal.

\section{Method}

\subsection{Study Design, Setting and Ethical Consideration}

This was a retrospective cross-sectional study conducted at KAHS-TH, Jumla from December 2017 to May 2020. Ethical approval was obtained from the ethical review board of KAHS-TH, Jumla. Written consent was obtained from each participant before sample collection.

\subsection{Study Population}

All participants were enrolled who visited the health care center for screening TB infection after receiving written consent and for children below 18 years of age, written consent was obtained from their respective guardians.

\subsection{Data Collection}

A standard chart was prepared for the collection of demographic data. The record of participants was cross-checked with the KAHS-TH record section to complement the collected data from the participants. The sample collected for GeneXpert test was tested for tuberculosis in KAHS-TH by experienced laboratory technologists. GeneXpert test reports were retrieved from the laboratory record book of KAHS-TH.

\subsection{Data Analysis}

After data cleaning using Excel, they were imported and analyzed using Statistical Package for the Social Sciences (SPSS) version 16.0.

\section{Results}

Altogether 805 GeneXpert test was done for the diagnosis of TB during the study period. Out of which 457 (57\%) were males and 348 (43\%) were females. (Figure 1)

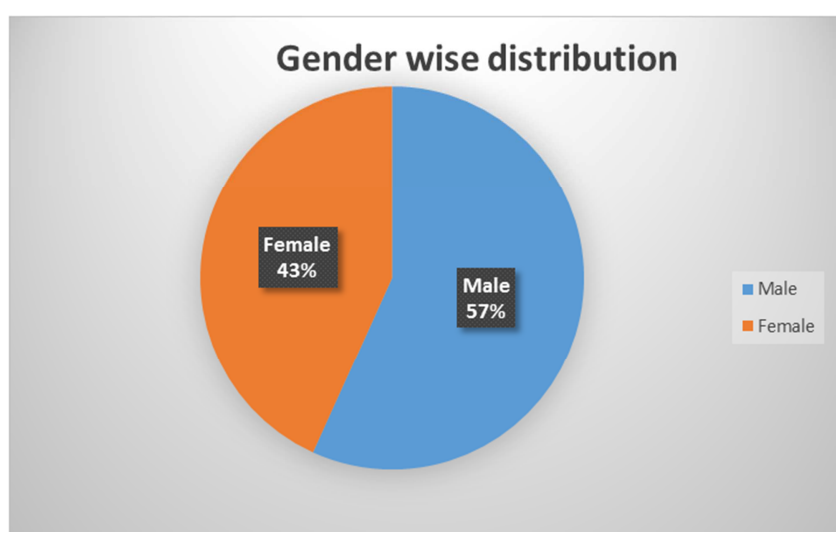

Figure 1. Gender-wise distribution of samples.

The age-wise distribution showed that the highest number of participants were found in the age group of 51-60 years i.e. $141(17.51 \%)$ followed by 61-70 years with $133(16.52 \%)$ which showed the involvement of older people in GeneXpert test for the diagnosis of TB.

Table 1. Age-wise distribution of male and female participants $(N=805)$.

\begin{tabular}{lllll}
\hline Age Groups in Year & Male & Female & Total & Percentage (\%) \\
\hline Under 10 & 25 & 12 & 37 & 4.59 \\
$11-20$ & 63 & 40 & 103 & 12.79 \\
$21-30$ & 63 & 63 & 126 & 15.65 \\
$31-40$ & 49 & 54 & 103 & 12.79 \\
$41-50$ & 54 & 52 & 106 & 13.16 \\
$51-60$ & 94 & 47 & 141 & 17.51 \\
$61-70$ & 71 & 62 & 133 & 16.52 \\
$71-80$ & 35 & 17 & 52 & 6.45 \\
Above 81 & 3 & 1 & 4 & 0.49 \\
Total & 457 & 348 & 805 & \\
\hline
\end{tabular}

The overall prevalence rate of TB was found to be $11.9 \%$ while $88.1 \%$ of participants were negative to TB infection. (Figure 2)

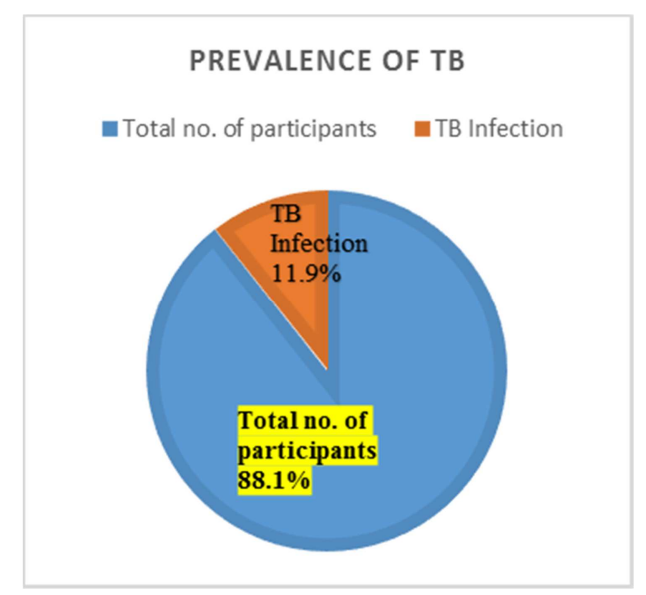

Figure 2. Prevalence of TB by GeneXpert method. 
Mostly sputum specimens were used for the diagnosis of TB infection during this study period. Out of 770 sputum specimens, total positive cases were 93 (12.07\%). Within these total cases, we found $89 \mathrm{~TB}$ positive cases and $4 \mathrm{~TB}$ positive with rifampicin-resistant cases. Similarly, there were a total of 6 fine needle aspirated specimens where we found 2 $(33.33 \%)$ TB positive cases and in 20 pleural fluid, only 1 $(5 \%)$ was TB positive case. However, TB positive case was not detected in any of ascitic fluid, peritoneal fluid, pericardial fluid, CSF, and urine specimens. (Table 2)

Table 2. Different types of specimens used for GeneXpert test and the number of MTB positive and Rifampicin resistant.

\begin{tabular}{|c|c|c|c|c|c|}
\hline S. N. & Specimen Type & Total no. of cases & MTB detected & MTB+RR detected & Total Positive N (\%) \\
\hline 1 & Sputum & 770 & 89 & 4 & $93(12.07 \%)$ \\
\hline 2 & Pleural fluid & 20 & 1 & 0 & $1(5 \%)$ \\
\hline 3 & Ascitic fluid & 3 & 0 & 0 & 0 \\
\hline 4 & Peritoneal fluid & 2 & 0 & 0 & 0 \\
\hline 5 & Pericardial fluid & 1 & 0 & 0 & 0 \\
\hline 6 & CSF & 1 & 0 & 0 & 0 \\
\hline 7 & Urine & 2 & 0 & 0 & 0 \\
\hline & Total & 805 & 92 & 4 & \\
\hline
\end{tabular}

Figure 3 illustrates the high prevalence rate of TB found in males, 67 (14.66\%) as compared to females, 29 (8.33\%) in which total of 4 cases of rifampicin-resistant was found, 2 cases on each gender.

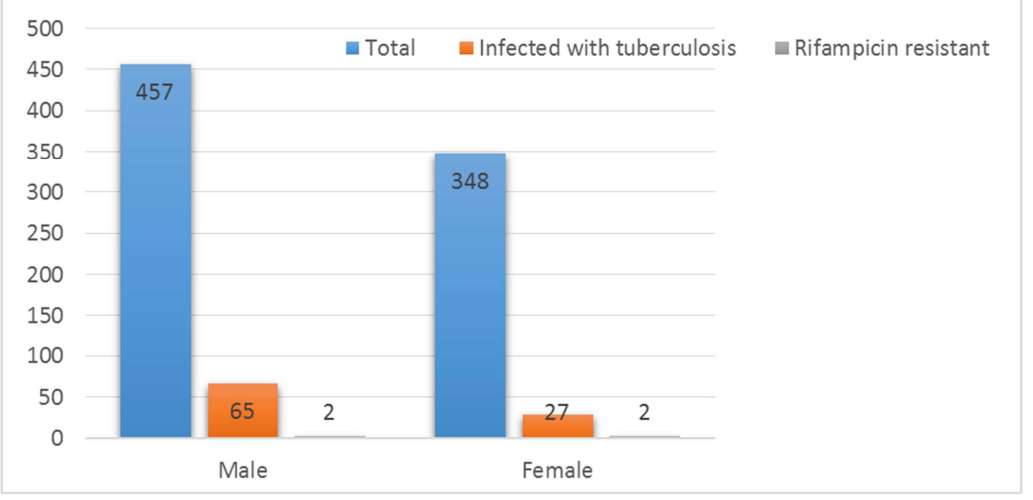

Figure 3. TB infection and rifampicin-resistant in male and female.

The highest prevalence of TB infection $26(20.63 \%)$ was found in the age group of 21-30 years whereas there was no prevalence above 81 years and very low i.e. 1 (2.70\%) was found under 10 years. (Table 3 )

Table 3. Prevalence of TB in males and females with age groups.

\begin{tabular}{|c|c|c|c|c|c|}
\hline \multirow{2}{*}{ Age Groups } & \multicolumn{2}{|l|}{ Male } & \multicolumn{2}{|c|}{ Female } & \multirow{2}{*}{ Total } \\
\hline & Total & Positive & Total & Positive & \\
\hline Under 10 & 25 & $1(4.0 \%)$ & 12 & 0 & $1(2.70 \%)$ \\
\hline $11-20$ & 63 & $4(6.3 \%)$ & 40 & $6(15.0 \%)$ & $10(9.70 \%)$ \\
\hline $21-30$ & 63 & $15(23.8 \%)$ & 63 & $11(17.5 \%)$ & $26(20.63 \%)$ \\
\hline $31-40$ & 49 & $9(18.3 \%)$ & 54 & $3(5.6 \%)$ & $12(11.65 \%)$ \\
\hline $41-50$ & 54 & $9(16.7 \%)$ & 52 & $3(5.8 \%)$ & $12(11.32 \%)$ \\
\hline $51-60$ & 94 & $11(11.7 \%)$ & 47 & 0 & $11(7.80 \%)$ \\
\hline $71-80$ & 35 & $6(17.1 \%)$ & 17 & $2(11.8 \%)$ & $8(15.38 \%)$ \\
\hline Above 81 & 3 & 0 & 1 & 0 & 0 \\
\hline Total & 457 & $67(14.66 \%)$ & 348 & $29(8.33 \%)$ & \\
\hline
\end{tabular}

\section{Discussion}

TB remains a major public health threat in Nepal and responsible for infecting thousands of people each year. The Government of Nepal recognizes the responsibility of contribution towards the global efforts to ending the TB epidemic by 2030 by improving accessibility and availability of quality TB services in the country. [8] Worldwide, TB is the top infectious disease as in 2018, 1.5 million people died from TB including 251000 deaths among people with HIV and a major cause of death was due to antimicrobial resistance. [4] According to the WHO, GeneXpert test is the most rapid and sensitive test for the diagnosis of $\mathrm{TB}$ in respiratory samples. [9]

In our study, we evaluated the impact of GeneXpert to find out TB diagnosis and treatment effectiveness among the individuals visiting KAHS-TH, Jumla. GeneXpert test was 
first started in KAHS-TH from $6^{\text {th }}$ December 2017, and only 805 tests were performed till $5^{\text {th }}$ May 2020, so the overall impact of this test was much less. In this study, we found only $11.9 \%$ prevalence of TB by the GeneXpert method in KAHS-TH, Jumla which is lower than other provinces of Nepal. [10] But higher as compared to other studies with a prevalence of $5.3 \%, 7.2 \%, 6.5 \%$. [11-13]

Out of 805 participants, 457 (57\%) males and 348 (43\%) females were tested for GeneXpert test. We found a high prevalence rate of $\mathrm{TB}$ infection in males (14.66\%) as compared to females $(8.33 \%)$ which was in agreement with a study conducted in the National Tuberculosis Center, Bhaktapur, Nepal (27.55\% male, 25\% female).[14] This reveals males are more prone to TB infection than females. This finding indicates the fact that males are more exposed to the outer environment than females, and also directly or indirectly there is a higher probability of them coming in contact with TB infected or suspected patients.

Rifampicin-resistant MTB isolates detected by GeneXpert were strong predictors of MDR-TB. [13] Although the numbers of MTB/RR i.e. $4(4.16 \%)$ in the present study were less than in similar studies in Ethiopia. $[15,16]$

In this study, the age group of 22-30 years showed the highest prevalence rate of $26(20.63 \%)$. This implies the young and adult working population was at high risk of being affected by TB. But there was no TB infection was in the age group above 80 years and less in the age group under 10 years.

Among the different specimens collected, sputum specimens were mostly used during the study period. MTB and MTB/RR were detected in $93(12.07 \%)$ sputum specimens, $2(33.33 \%)$ cases are only MTB positive in fine needle aspirated specimens, and $1(5 \%)$ case was MTB positive in pleural fluid. Though there were no MTB positive in ascetic fluid, peritoneal fluid, pericardial fluid, cerebrospinal fluid, and urine sample. The reason may be in these specimens there were low bacterial concentrations.

The National Strategic Plan (NSP) set ambitious targets to reduce the incidence of TB by $20 \%$ by 2021 . Also, increase diagnosis and treatment gaps of MDR/RR-TB (multidrug-resistant $\mathrm{TB}$ and rifampicin-resistant $\mathrm{TB}$ ) and confirm treatment success rate reached and maintained above $90 \%$ for drug-sensitive (DS) tuberculosis and $75 \%$ for DR/RR-TB cases. The NSP also predicts improved drug-susceptible testing coverage and improved access to newer diagnostics and newer TB drugs where required. [17]

Nevertheless, there are some limitations of this study. First, it was held in a single health institute with a small sample size of suspected TB. It would be better if we involve many health institutions with a large number of populations for a better demonstration of the study subjects. Second, this study relied only on a single testing method i.e. GeneXpert and lacks other diagnostic tools that can be used as comparators like sputum smear, culture, chest X-ray results. Also, the patients with smear-positive for TB may be missed thus underestimating the total prevalence. Third, there were only limited numbers of variables for analysis. At last, the absence of rpoB mutations does not rule out MDR-TB.

Nonetheless, this study provides a fair estimate of the prevalence of tuberculosis in this rural region of Nepal which may help for future strategy and planning to be employed to meet the target set by the NSP for TB prevention, care, and control (2016- 2021). A future study using a generalized random sampling method may give a complete estimate of regional and national prevalence rates.

\section{Conclusion}

This study provides preliminary information on the overall prevalence of TB infection in Jumla, Nepal. GeneXpert test helps to increase $\mathrm{TB}$ case detection as well as rifampicin-resistant which ultimately helps in treatment and control of TB. Although our sample size was small, the actual impact of GeneXpert on diagnosis and initiation of $\mathrm{TB}$ treatment further emphasizes the need to identify strategies to maximize and cost-effectiveness of GeneXpert test. This may help to meet the national strategies set by the national tuberculosis control program. The finding slightly differs from the little available data on TB in the region but may be relevant to similar settings. These results have also identified groups of people with the highest risk of TB infection in Jumla, Nepal. More and descriptive studies should be conducted to prioritize prevention, treatment, and immunization in the future.

\section{Conflict of Interests}

The authors declare that they have no competing interests.

\section{Acknowledgements}

We would like to acknowledge the Institutional Review Committee (IRC) of Karnali Academy of Health Sciences Teaching Hospital (KAHS-TH). We would also like to acknowledge the laboratory staffs of KAHS-TH. At last special thanks to those who participated in the study.

\section{References}

[1] World Health Organisation. (2016) Chest Radiography in Tuberculosis. WHO Library Cataloguing-in-Publication Data, 44. https://doi.org/ISBN 9789241511506.

[2] (WHO), W.H.O. Tuberculosis Fact Sheet.

[3] World Health Organization; Licence: CC BY-NCcitation. Global tuberculosis report 2017. Geneva: World Health Organization; 2017. Licence: CC BY-NCSA and 3.0 IGO. (2017) Global tuberculosis report 2017. Geneva: World Health Organization; 2017.

[4] WHO. (2019) WHO TB Report. WHO Library Cataloguing-in-Publication Data World, 7.

[5] World Health Organization (WHO). Millions more with Tuberculosis (TB) diagnosed and treated. 
[6] Center of Disease Control. (2013) A New Tool to Diagnose Tuberculosis: The Xpert MTB / RIF Assay. Cdc, 2. https://doi.org/10.1016/j.soilbio.2014.08.009.

[7] Boehme, C. C., Nabeta, P., Hillemann, D., Nicol, M. P., Shenai, S., Krapp, F. et al. (2010) Rapid Molecular Detection of Tuberculosis and Rifampin Resistance. Noticias Jurídicas, 363, 1005-15. https://doi.org/10.1056/NEJMoa0907847.

[8] World Health Organization. (2018) Global tuberculosis report 2018. World Health Organization. http://www.who.int/iris/handle/10665/274453.

[9] Organization, W. H. (2011) Automated real-time nucleic acid amplification technology for simultaneous and rapid detection of tuberculosis and rifampicin resistance: Xpert MTB/RIF. Policy Statement WHO/HTM/TB/20114 Geneva.

[10] Government of Nepal Ministry of Health and Population. (2018) Annual Report 2018, National Tuberculosis Program, Nepal. Nepal Tuberculosis Center, Nepal, 75, 1-106. https://doi.org/10.1017/CBO9781107415324.004.

[11] Gebrecherkos, T., Gelaw, B. and Tessema, B. (2016) Smear positive pulmonary tuberculosis and HIV co-infection in prison settings of North Gondar Zone, Northwest Ethiopia. BMC Public Health, BMC Public Health. 16, 1-10. https://doi.org/10.1186/s12889-016-3761-y.

[12] Mama, M., Manilal, A., Tesfa, H., Mohammed, H. and Erbo, E. (2018) Prevalence of Pulmonary Tuberculosis and Associated Factors Among HIV Positive Patients Attending Antiretroviral Therapy Clinic at Arba Minch General Hospital, Southern
Ethiopia. The Open Microbiology Journal, 12, 163-71. https://doi.org/10.2174/1874285801812010163.

[13] Sinshaw, W., Kebede, A., Bitew, A., Tesfaye, E., Tadesse, M., Mehamed, Z. et al. (2019) Prevalence of tuberculosis, multidrug resistant tuberculosis and associated risk factors among smear negative presumptive pulmonary tuberculosis patients in Addis Ababa, Ethiopia. BMC Infectious Diseases, BMC Infectious Diseases. 19, 1-15. https://doi.org/10.1186/s12879-019-4241-7.

[14] Thapa, A., Gurung, P. and Ghimire, G. R. (2017) Evaluation of Gene Xpert Mtb/Rif Assay for the Detection of Mycobacterium Tuberculosis in Sputum of Patients Suspected of Pulmonary Tuberculosis Visiting National Tuberculosis Centre, Thimi, Bhaktapur, Nepal. SAARC Journal of Tuberculosis, Lung Diseases and HIV/AIDS, 13, 16-22. https://doi.org/10.3126/saarctb.v13i1.16924.

[15] Jaleta, K. N., Gizachew, M., Gelaw, B., Tesfa, H., Getaneh, A. and Biadgo, B. (2017) Rifampicin-resistant Mycobacterium tuberculosis among tuberculosis-presumptive cases at University of Gondar Hospital, northwest Ethiopia. 185-92.

[16] Arega, B., Menbere, F. and Getachew, Y. (2019) Prevalence of rifampicin resistant Mycobacterium tuberculosis among presumptive tuberculosis patients in selected governmental hospitals in Addis Ababa, Ethiopia. BMC Infectious Diseases, BMC Infectious Diseases. 19, 1-5. https://doi.org/10.1186/s12879-019-3943-1.

[17] NTC. (2016) National Strategic Plan for Tuberculosis Prevention, Care and Control, 2016-2021. 36. 